TRANSACTIONS OF THE

AMERICAN MATHEMATICAL SOCIETY

Volume 362, Number 10, October 2010, Pages 5355-5371

S 0002-9947(2010)04919-0

Article electronically published on May 19, 2010

\title{
NUMEROSITIES OF POINT SETS OVER THE REAL LINE
}

\author{
MAURO DI NASSO AND MARCO FORTI
}

\begin{abstract}
We consider the possibility of a notion of size for point sets, i.e. subsets of the Euclidean spaces $\mathbb{E}_{d}(\mathbb{R})$ of all $d$-tuples of real numbers, that satisfies the fifth common notion of Euclid's Elements: "the whole is larger than the part". Clearly, such a notion of "numerosity" can agree with cardinality only for finite sets. We show that "numerosities" can be assigned to every point set in such a way that the natural Cantorian definitions of the arithmetical operations provide a very good algebraic structure. Contrasting with cardinal arithmetic, numerosities can be taken as (nonnegative) elements of a discretely ordered ring, where sums and products correspond to disjoint unions and Cartesian products, respectively. Actually, our numerosities form suitable semirings of hyperintegers of nonstandard Analysis. Under mild set-theoretic hypotheses $\left(e . g \cdot \operatorname{cov}(\mathcal{B})=\mathfrak{c}<\aleph_{\omega}\right)$, we can also have the natural ordering property that, given any two countable point sets, one is equinumerous to a subset of the other. Extending this property to uncountable sets seems to be a difficult problem.
\end{abstract}

\section{INTRODUCTION}

Historically, the Cantorian theory of cardinalities originates from the idea of extending the notion of counting from finite sets to arbitrary "Punktmengen", i.e. arbitrary subsets of the Euclidean space $\mathbb{E}_{d}(\mathbb{R})$ of dimension $d$; see [8]. Cardinal numbers were defined according to the natural "counting principle" that two point sets have the same "number of elements" if and only if they can be put in one-to-one correspondence. Cantor extended sum, product, and ordering of integers to infinite cardinals by assuming the following natural principles $(6,6])$ :

(C.1): If $A$ is a finite set with $n$ points, then $|A|=n$.

(C.2): $|A|+|B|=|A \cup B|$ provided $A \cap B=\emptyset$.

(C.3): $|A| \cdot|B|=|A \times B|$ for all $A, B$.

(C.4): $|A| \leq|B|$ if and only if there exists $C \subseteq B$ such that $|A|=|C|$.

In the Cantorian theory of cardinalities these properties give rise to a very weird arithmetic, where

$$
\mathfrak{a}+\mathfrak{b}=\mathfrak{a} \cdot \mathfrak{b}=\max \{\mathfrak{a}, \mathfrak{b}\} \text { for all infinite } \mathfrak{a}, \mathfrak{b} .
$$

Our purpose is to give a new way of counting infinite collections, so as to obtain a satisfactory arithmetic for the corresponding "numerosities", while maintaining the Cantorian properties above. In particular, we want that our (nonzero) numerosities are contained in the positive part of a discretely ordered ring. As a consequence,

Received by the editors July 25, 2008.

2010 Mathematics Subject Classification. Primary 03E65, 03E05; Secondary 03E35, 03A05, $03 \mathrm{C} 20$.

(C)2010 American Mathematical Society Reverts to public domain 28 years from publication 
having the same numerosity is no more equivalent to being in 1-1 correspondence, for infinite sets. In fact our notion of equinumerosity can be viewed as a kind of proper refinement of the notion of equipotency, where the Cantorian weak ordering principle (C.4) is strengthened by considering strict inequality and proper inclusion. The "if part" of the resulting principle (ord) of Definition1.1 below, incorporates the famous fifth Euclidean common notion: "the whole is greater than the part". On the other hand, as a consequence of the "only if" part, the ordering of numerosities is intrinsically defined in terms of subsets, as it is in the case of cardinalities, instead of being simply inherited from the given ordered ring.

This work is part of a research programme jointly developed by Vieri Benci and the authors in the last half-decade. In [1, a first notion of numerosity is axiomatically introduced for "labelled sets", so as to satisfy the "Zermelian" property that given any two labelled sets one is equinumerous to a subset of the other. Labelled sets are sets equipped with a finite-to-one labelling by natural numbers, and so they are necessarily countable. Moreover their numerosities essentially depend on the labelling. For the existence of such numerosities the use of Ramsey ultrafilters is essential. A notion of numerosity for arbitrary sets of ordinals was first considered in [2, where the "Cantorian" property that equinumerous sets are equipotent is assumed. The corresponding models make use of special ultrafilters over the sets of finite parts of cardinals. Finally, in [3, numerosity functions are defined on all sets of (sufficiently small) "mathematical" universes, i.e. superstructures $V(X)=\bigcup_{n \in \mathbb{N}} V_{n}(X)$ over a base set $X$ of size less than $\aleph_{\omega}$. In particular, under mild set-theoretic hypotheses, a given numerosity function for countable sets can be extended to the whole universe, in such a way that numerosities are continuous with respect to "normal approximations" 1

Similarly to the original Cantorian work [8, 10, we focus here on point sets, where a $\left(d\right.$-dimensional) point set is any subset of the Euclidean space $\mathbb{E}_{d}(\mathbb{R})=\mathbb{R}^{d}$ of all $d$-tuples of real numbers. The restriction to finite-dimensional point sets is useful in view of the fact, extensively illustrated in [2, that the product principle (C.3) postulated for all sets is inconsistent with the fifth Euclidean common notion 2 On the other hand, we remark that the geometric structure of the Euclidean space is irrelevant in this context. In fact even translations and rotations may not preserve numerosities, as a consequence of the Banach-Tarski paradox. The only thing that matters here is the cardinality of the line $\mathbb{R}$.

The paper is organized as follows. In Section 1 we introduce the explicit definition of a (Cantorian, Zermelian, and normal) numerosity function for point sets, and we state our main result. In Section 2 we assume the Continuum Hypothesis and we show that there exist Zermelian numerosity functions on all countable point sets, having (a very special kind of) nonstandard integers as numerosities. By suitably enlarging these rings of nonstandard integers, we extend these numerosity functions to normal numerosity functions defined on all point sets. In Section 3 we show that weaker set-theoretic assumptions, allowing for an arbitrary size of the Continuum, still suffice to define Cantorian numerosity functions on all point sets, which are Zermelian when restricted to countable point sets. Under slightly

\footnotetext{
${ }^{1}$ For a precise definition of these notions, see Definition 1.2 below.

${ }^{2} E$.g. if $A=\{0,(0,0),(0,0,0), \ldots\}$, then $\{0\} \times A$ is a proper subset of $A$, and so $1 \cdot \mathfrak{n}(A)$ should be less than $\mathfrak{n}(A)$.
} 
stronger set-theoretic hypotheses $\left(e \cdot g \cdot \operatorname{cov}(\mathcal{B})=\mathfrak{c}<\aleph_{\omega}\right) 3$ we show that there exist normal numerosity functions on all point sets that are Zermelian on all countable sets. The question as to whether there exist Zermelian numerosity functions on uncountable point sets will be considered in the last section, Section 4, where other final remarks and open questions can be found.

In general, we refer to 12 for all the set-theoretical notions and facts used in this paper, and to 9 for definitions and facts concerning ultrapowers, ultrafilters, and nonstandard models.

The authors are grateful to V. Benci, K. Hrbacek, and M. Viale for some discussions on the subject, to A. Blass for several useful remarks about ultrafilters, and to the referee for some useful suggestions.

\section{Numerosity FunCtions}

We define a ( $d$-dimensional) point set to be any subset of the Euclidean space $\mathbb{E}_{d}(\mathbb{R})=\mathbb{R}^{d}$ of all $d$-tuples of real numbers. As usual in the mathematical praxis, we identify the Cartesian product $\mathbb{E}_{d}(\mathbb{R}) \times \mathbb{E}_{k}(\mathbb{R})$ with the $(d+k)$-dimensional space $\mathbb{E}_{d+k}(\mathbb{R})$. Throughout the paper, we let

$$
\mathbb{W}=\bigcup_{d \in \mathbb{N}} \mathcal{P}\left(\mathbb{E}_{d}(\mathbb{R})\right) \quad \text { and } \quad \mathbb{W}_{0}=\bigcup_{d \in \mathbb{N}}\left[\mathbb{E}_{d}(\mathbb{R})\right] \leq \aleph_{0}
$$

be the set of all point sets and the set of all countable point sets, respectively.

We introduce the notion of numerosity as a sort of "measure" for point sets, taking values in a discretely ordered ring and incorporating the five classical Euclidean Common Notions on magnitudes. In a different context, numerosities satisfying similar properties have been introduced in [2, 3. We phrase our main definition as follows:

Definition 1.1. Let $\mathfrak{n}$ be a function defined on point sets and taking values in the nonnegative part of a discretely ordered ring. We say that $\mathfrak{n}$ is a numerosity function if the following conditions are fulfilled 4

(unit): $\mathfrak{n}(\{x\})=1$ for every point $x$.

(sum): $\mathfrak{n}(X)+\mathfrak{n}(Y)=\mathfrak{n}(X \cup Y)$ provided $X \cap Y=\emptyset$.

(prod): $\mathfrak{n}(X) \cdot \mathfrak{n}(Y)=\mathfrak{n}(X \times Y)$ for all $X, Y$.

We say that the numerosity function $\mathfrak{n}$ is Zermelian if

(ord): $\mathfrak{n}(X)<\mathfrak{n}(Y)$ if and only if there exists $Z \subset Y$ such that $\mathfrak{n}(X)=\mathfrak{n}(Z)$.

We say that the numerosity function $\mathfrak{n}$ is Cantorian if

(cant): $|X|<|Y| \Longrightarrow \mathfrak{n}(X)<\mathfrak{n}(Y)$.

The range $\mathfrak{N}$ of $\mathfrak{n}$ is called the set of numerosities.

The adjective 'Zermelian' has been chosen because, for a numerosity function, the property (ord) is equivalent to the following "Zermelian" property:

- Given two sets, one has the same numerosity as a subset of the other.

By assuming mild set-theoretic hypotheses, we shall show that there exist Cantorian numerosity functions on all point sets that are Zermelian on all countable

\footnotetext{
3 For cardinals of the continuum we adhere to the notation of 4 .

4 We implicitly assume that the conditions apply provided that the involved point sets belong to the domain of $\mathfrak{n}$.
} 
sets. The question as to whether there exist Zermelian numerosity functions on uncountable point sets will be considered in the last section.

Any set can be viewed as "approximable" by subsets of smaller cardinality. So, in order to also measure uncountable point sets, one might require that the numerosity function be continuous with respect to appropriately chosen approximations. With this in mind, we report here for convenience the notion of normal approximation as given in [3]:

Definition 1.2. Let $\kappa$ be an uncountable cardinal. The $\kappa$-sequence $\left\langle X_{\alpha} \mid \alpha<\kappa\right\rangle$ is a normal approximation of the set $X$ if the following conditions are fulfilled:

(1) $\alpha<\beta<\kappa \Longrightarrow X_{\alpha} \subseteq X_{\beta}$;

(2) $X_{\lambda}=\bigcup_{\alpha<\lambda} X_{\alpha}$ for all limits $\lambda<\kappa$;

(3) $\left|X_{\alpha}\right|<|X|$ for all $\alpha<\kappa$;

(4) $X=\bigcup_{\alpha<\kappa} X_{\alpha}$.

A numerosity function $\mathfrak{n}$ is normal if

$$
\mathfrak{n}\left(X_{\alpha}\right) \leq \mathfrak{n}\left(Y_{\alpha}\right) \text { for all } \alpha<\kappa \Longrightarrow \mathfrak{n}(X) \leq \mathfrak{n}(Y)
$$

whenever $\left\langle X_{\alpha} \mid \alpha<\kappa\right\rangle$ and $\left\langle Y_{\alpha} \mid \alpha<\kappa\right\rangle$ are normal approximations of $X$ and $Y$, respectively.

It is easily verified (see [3]) that if $|X|$ is regular uncountable, then any two normal approximations of $X$ are equivalent in the sense that they have the same length $\kappa=|X|$ and they agree on a closed unbounded subse 5 of $\kappa$.

The main results of this paper can be summarized as follows:

Main Theorem. Assume that $\mathfrak{c}<\aleph_{\omega}$, and let $\mathcal{U}$ be a nonprincipal ultrafilter on $\mathbb{N}$. Then there exists a numerosity function defined on all countable point sets $\mathfrak{n}_{\mathcal{U}}: \mathbb{W}_{0} \rightarrow \mathbb{Z}_{\mathcal{U}}^{\mathbb{N}}$, which is Zermelian whenever $\mathcal{U}$ is Ramsey.

Moreover there exist ultrafilters $\mathcal{V}, \mathcal{W}$ over $\mathbb{R}$ such that $\mathfrak{n}_{\mathcal{U}}$ can be extended to numerosity functions $\mathfrak{n}_{\mathcal{V}}: \mathbb{W} \rightarrow \mathbb{Z}_{\mathcal{V}}^{\mathbb{R}}$ and $\mathfrak{n}_{\mathcal{W}}: \mathbb{W} \rightarrow \mathbb{Z}_{\mathcal{W}}^{\mathbb{R}}$ that are, respectively, normal and Cantorian. So in each case the set of numerosities can be taken to be a semiring of nonnegative hyperintegers.

Recall that a nonprincipal ultrafilter $\mathcal{U}$ on a countable set $I$ is called Ramsey (or selective) if for every partition $\left\{A_{n} \mid n \in \mathbb{N}\right\}$ of $I$ either there exists $n$ such that $A_{n} \in \mathcal{U}$ or there exists $U \in \mathcal{U}$ such that $\left|A_{n} \cap U\right| \leq 1$ for all $n$. Many equivalent properties can be given; see e.g. [1. The most relevant for our purposes is the following one: a nonprincipal ultrafilter $\mathcal{U}$ on $\mathbb{N}$ is Ramsey if and only if every function $f: \mathbb{N} \rightarrow \mathbb{N}$ is $\mathcal{U}$-equivalent to a nondecreasing one.

\section{Measuring point Sets under the Continuum Hypothesis}

Throughout this section we assume the Continuum Hypothesis $\mathrm{CH}$, i.e. $\mathfrak{c}=\omega_{1}$. So the real numbers are the increasing union of an $\omega_{1}$-chain of countable subsets. As any countable set $A$ is the increasing union of a chain of finite subsets $A_{n}$, every subset $X$ of $A$ can be "counted" by considering the sequence of nonnegative integers

\footnotetext{
${ }^{5}$ Recall that a subset $X \subseteq \kappa$ is unbounded in $\kappa$ if $\sup X=\kappa$, and $X$ is closed if $\sup A \in X$ for all $A \subseteq X$ that are bounded in $\kappa$. If $\kappa$ is regular uncountable, then the closed unbounded sets generate a ( $\kappa$-additive) filter $\mathcal{F}_{\kappa}$ called the closed unbounded filter (shortly the club filter) on $\kappa$ (see 12]).
} 
$\left|X \cap A_{n}\right|$. In the following subsection we show how to coherently combine the above remarks so as to obtain coherent finite approximations of the real line.

2.1. Finite approximations of the real line. In this subsection we find appropriate families of finite subsets of $\mathbb{R}$, suitable for providing a "counting process" for all countable point sets.

Lemma 2.1. Assume $\mathrm{CH}$, and let $\left\langle r_{\alpha} \mid \alpha<\omega_{1}\right\rangle$ be an enumeration of $\mathbb{R}$. For $\alpha<\omega_{1}$ put $\mathbb{R}^{\alpha}=\left\{r_{\beta} \mid \beta<\alpha\right\}$. Then, for all $\alpha<\omega_{1}$ and all $n \in \mathbb{N}$ there exist finite sets $\mathbb{R}_{n}^{\alpha}$ and functions $\varphi_{\alpha}: \alpha \rightarrow \mathbb{N}$ such that

(i): $\mathbb{R}_{n}^{\alpha} \subset \mathbb{R}_{n+1}^{\alpha}$ and $\bigcup_{n \in \mathbb{N}} \mathbb{R}_{n}^{\alpha}=\mathbb{R}^{\alpha}$;

(ii): if $\beta<\alpha$ and $n \geq \varphi_{\alpha}(\beta)$, then $\mathbb{R}_{n}^{\beta}=\mathbb{R}_{n}^{\alpha} \cap \mathbb{R}^{\beta}$.

Proof. We proceed by induction on $\alpha$. The basis $\alpha=0$ is trivial. Assume that $\mathbb{R}_{n}^{\beta}$ and $\varphi_{\beta}$ are defined for all $\beta<\alpha$ so as to fulfill conditions (i) and (ii).

If $\alpha=\gamma+1$ is a successor ordinal, the desired conditions are satisfied by putting

$$
\mathbb{R}_{n}^{\alpha}=\mathbb{R}_{n}^{\gamma} \cup\left\{r_{\gamma}\right\} \text { and } \varphi_{\alpha}(\beta)= \begin{cases}\varphi_{\gamma}(\beta) & \text { if } \beta<\gamma, \\ 0 & \text { if } \beta=\gamma\end{cases}
$$

If $\alpha$ is a limit ordinal, fix an enumeration $\left\langle\alpha_{i} \mid i \in \mathbb{N}\right\rangle$ of $\alpha$ and put

$$
g(k)=\max \left\{h \leq k \mid \forall i, j \leq h\left(\alpha_{i}<\alpha_{j} \Longrightarrow \alpha_{j} \leq \alpha_{h} \& \varphi_{\alpha_{j}}\left(\alpha_{i}\right) \leq k\right)\right\} .
$$

The function $g$ is nondecreasing by definition. We show that $g$ is unbounded. To this aim we define inductively the sequence of indices $\ell_{n}$ by putting $\ell_{0}=0$ and $\ell_{n+1}=\min \left\{i \in \mathbb{N} \mid \alpha_{i}>\alpha_{\ell_{n}}\right\}$. So the ordinal sequence $\alpha_{\ell_{n}}$ is increasing, and $i<\ell_{n}$ implies $\alpha_{i}<\alpha_{\ell_{n}}$. It follows that the values of $g(k)$ are chosen among the $\ell_{n}$ s. Given $m \in \mathbb{N}$, pick $n$ such that $\ell_{n} \geq m$ and put

$$
k_{n}=\max \left\{\varphi_{\alpha_{j}}\left(\alpha_{i}\right) \mid i, j \leq \ell_{n}, \alpha_{i}<\alpha_{j}\right\} .
$$

If $k_{n} \geq \ell_{n}$, then $g\left(k_{n}\right) \geq \ell_{n}$. If $k_{n} \leq \ell_{n}$, then $g\left(\ell_{n}\right)=\ell_{n}$. So in both cases $g\left(\max \left\{k_{n}, \ell_{n}\right\}\right) \geq \ell_{n} \geq m$, and $g$ is unbounded. As a consequence, the sequence of ordinals $\beta_{n}=\alpha_{g(n)}$ is nondecreasing and unbounded in $\alpha$.

Now put $\mathbb{R}_{n}^{\alpha}=\mathbb{R}_{n}^{\beta_{n}}$, so

$$
\mathbb{R}_{n}^{\alpha} \cap \mathbb{R}^{\alpha_{i}}=\mathbb{R}_{n}^{\beta_{n}} \cap \mathbb{R}^{\alpha_{i}}=\mathbb{R}_{n}^{\alpha_{i}} \text { whenever } \alpha_{i}<\beta_{n} \text { and } n \geq \varphi_{\beta_{n}}\left(\alpha_{i}\right) .
$$

Define the function $\varphi_{\alpha}$ by

$$
\varphi_{\alpha}\left(\alpha_{i}\right)=\min \{n \in \mathbb{N} \mid i<g(n)\} .
$$

Then $n \geq \varphi_{\alpha}\left(\alpha_{i}\right)$ implies both $\alpha_{i}<\alpha_{g(n)}=\beta_{n}$ and $n \geq \varphi_{\beta_{n}}\left(\alpha_{i}\right)$, and condition (ii) is fulfilled. Moreover one has

$$
\mathbb{R}_{n+1}^{\alpha}=\mathbb{R}_{n+1}^{\beta_{n+1}} \supseteq \mathbb{R}_{n+1}^{\beta_{n+1}} \cap \mathbb{R}^{\beta_{n}}=\mathbb{R}_{n+1}^{\beta_{n}} \supseteq \mathbb{R}_{n}^{\beta_{n}}=\mathbb{R}_{n}^{\alpha}
$$

because $n+1 \geq \varphi_{\beta_{n+1}}\left(\beta_{n}\right)$, and the first clause of (i) follows.

Finally, given $r \in \mathbb{R}^{\alpha}$, let $i, m$ be such that $r \in \mathbb{R}_{m}^{\alpha_{i}}$, and pick $n \geq m$ such that $g(n) \geq i$. Then

$$
r \in \mathbb{R}_{m}^{\alpha_{i}} \subseteq \mathbb{R}_{n}^{\alpha_{i}}=\mathbb{R}_{n}^{\beta_{n}} \cap \mathbb{R}^{\alpha_{i}} \subseteq \mathbb{R}_{n}^{\alpha}
$$

because $n \geq \varphi_{\beta_{n}}\left(\alpha_{i}\right)$, and also the second clause of (i) is proved. 
Observe that we have wide freedom in choosing the approximating sequence in the case of additively decomposable limit ordinals 6 E.g., we can arbitrarily deal with successor limits, namely

Remark 2.2. For each limit ordinal $\alpha<\omega_{1}$, let $\left\langle a_{n}^{\alpha} \mid n \in \mathbb{N}\right\rangle$ be an unbounded nondecreasing sequence of natural numbers. Then the sequences $\left\langle\mathbb{R}_{n}^{\alpha+\omega} \mid n \in \mathbb{N}\right\rangle$ can be chosen in such a way that

$$
\mathbb{R}_{n}^{\alpha+\omega}=\mathbb{R}_{n}^{\alpha} \cup A_{n}^{\alpha}, \text { where }\left|A_{n}^{\alpha}\right|=a_{n}^{\alpha}
$$

for all limit ordinals $\alpha<\omega_{1}$.

In fact one can simply take $A_{n}^{\alpha}=\left\{r_{\alpha}, \ldots, r_{\alpha+a_{n}^{\alpha}-1}\right\}$.

2.2. Measuring countable point sets. We are now ready to give numerosity functions for all countable (finite-dimensional) point sets.

Lemma $2.3(\mathrm{CH})$. Given a point set $X \subseteq \mathbb{E}_{d}(\mathbb{R})$ put

$$
X_{n}^{\alpha}=X \cap\left(\mathbb{R}_{n}^{\alpha}\right)^{d} \text { for } \alpha<\omega_{1} \text { and } n \in \mathbb{N} \text {. }
$$

Then we have

(a): $\forall x \in\left(\mathbb{R}^{\alpha}\right)^{d} \exists m \forall n \geq m \quad\{x\}_{n}^{\alpha}=\{x\}$;

(b): $\forall X, Y \subseteq\left(\mathbb{R}^{\alpha}\right)^{d} \forall n(X \cup Y)_{n}^{\alpha}=X_{n}^{\alpha} \cup Y_{n}^{\alpha}$;

(c): $\forall X \subseteq\left(\mathbb{R}^{\alpha}\right)^{d} \forall Y \subseteq\left(\mathbb{R}^{\alpha}\right)^{d^{\prime}} \forall n \quad(X \times Y)_{n}^{\alpha}=X_{n}^{\alpha} \times Y_{n}^{\alpha}$;

(d): $\forall \beta>\alpha \exists m \forall n \geq m \forall X \subseteq\left(\mathbb{R}^{\alpha}\right)^{d} X_{n}^{\alpha}=X_{n}^{\beta}$.

Proof. Let $x=\left(x_{1}, \ldots, x_{d}\right) \in\left(\mathbb{R}^{\alpha}\right)^{d}$. Pick $m$ such that $x_{i} \in \mathbb{R}_{m}^{\alpha}$ for all $1 \leq i \leq d$. Then clearly $x \in\left(\mathbb{R}_{n}^{\alpha}\right)^{d}$ for all $n \geq m$, and (a) is proved.

Properties (b) and (c) are immediate consequences of the definitions.

Finally, using the notation of Lemma 2.1. property (d) is fulfilled by taking $m=\varphi_{\beta}(\alpha)$.

Let $\mathcal{F}$ be a filter on $\mathbb{N}$ extending the Fréchet filter of the cofinite subsets of $\mathbb{N}$. Denote by $\mathbb{Z}_{\mathcal{F}}^{\mathbb{N}}$ the reduced power of $\mathbb{Z}$ modulo $\mathcal{F}$, i.e. the set of the equivalence classes $[z]_{\mathcal{F}}$ of all sequences of integers $z=\left\langle z_{n}: n \in \mathbb{N}\right\rangle$ modulo the $\mathcal{F}$-equivalence

$$
z \approx_{\mathcal{F}} w \Longleftrightarrow\left\{n \mid z_{n}=w_{n}\right\} \in \mathcal{F},
$$

which is a partially ordered ring.

Recall that we denote by $\mathbb{W}_{0}$ the set $\bigcup_{d \in \mathbb{N}}\left[\mathbb{E}_{d}(\mathbb{R})\right]^{\leq \aleph_{0}}$ of all countable (finitedimensional) point sets.

Theorem 2.4 $(\mathrm{CH})$. Define the function $\mathfrak{n}_{\mathcal{F}}: \mathbb{W}_{0} \rightarrow \mathbb{Z}^{\mathbb{N}}$ by

$$
\mathfrak{n}_{\mathcal{F}}(X)=\left[\left\langle\left|X_{n}^{\alpha}\right|: n \in \mathbb{N}\right\rangle\right]_{\mathcal{F}}
$$

where $\alpha$ is any countable ordinal such that $X \subseteq\left(\mathbb{R}^{\alpha}\right)^{d}$. Then

(1) $\mathfrak{n}_{\mathcal{F}}(X)=|X|$ for all finite $X, 7$

(2) $\mathfrak{n}_{\mathcal{F}}(X)+\mathfrak{n}_{\mathcal{F}}(Y)=\mathfrak{n}_{\mathcal{F}}(X \cup Y)+\mathfrak{n}_{\mathcal{F}}(X \cap Y)$;

(3) $\mathfrak{n}_{\mathcal{F}}(X) \cdot \mathfrak{n}_{\mathcal{F}}(Y)=\mathfrak{n}_{\mathcal{F}}(X \times Y)$;

(4) $X \subset Y \Longrightarrow \mathfrak{n}_{\mathcal{F}}(X)<\mathfrak{n}_{\mathcal{F}}(Y)$.

Hence the range $\mathfrak{N}$ of $\mathfrak{n}_{\mathcal{F}}$ is a positive subsemiring of the partially ordered ring $\mathbb{Z}_{\mathcal{F}}^{\mathbb{N}}$, and the function $\mathfrak{n}_{\mathcal{F}}$ satisfies the conditions (unit), (sum), and (prod) of a numerosity function, together with the "if part" of (ord).

${ }^{6}$ Recall that an ordinal $\alpha$ is additively decomposable if $\alpha=\beta+\gamma$ with $\beta, \gamma<\alpha$.

7 As usual, we identify the integers with the $\mathcal{F}$-equivalence classes of the constant sequences. 
Proof. First of all, observe that the function $\mathfrak{n}_{\mathcal{F}}$ is well defined, by Property (d) of Lemma 2.3

Points 2 and 3 are immediate consequences of the Properties (b) and (c) of the same lemma.

Finally, Point 1 follows by finite additivity from Property (a), and Point 4 follows by combining 1 and 2 .

Note that the ordering induced on the range $\mathfrak{N}$ of $\mathfrak{n}_{\mathcal{F}}$ by the natural partial order of $\mathbb{Z}^{\mathbb{N}}$ is linear if and only if $\mathcal{F}$ is an ultrafilter. However, in general, this ordering does not satisfy the full property (ord). On the other hand, the condition (ord) might be used as the definition of a (partial) order that is compatible with (but in general weaker than) the one induced by $\mathbb{Z}^{\mathbb{N}}$. Under the Continuum Hypothesis, we can find suitable ultrafilters where these orderings agree.

Let $\mathcal{U}$ be a nonprincipal ultrafilter on $\mathbb{N}$, and let $\mathfrak{n}_{\mathcal{U}}: \mathbb{W}_{0} \rightarrow \mathbb{Z}_{\mathcal{U}}^{\mathbb{N}}$ be the function defined as in Theorem 2.4. Then

Theorem 2.5 $(\mathrm{CH})$. The function $\mathfrak{n}=\mathfrak{n}_{\mathcal{U}}$ is a numerosity function defined on all countable point sets, which is Zermelian whenever the ultrafilter $\mathcal{U}$ is Ramsey.

Proof. The filter $\mathcal{U}$ being ultra, the natural order of $\mathbb{Z}_{\mathcal{U}}^{\mathbb{N}}$ is linear. So, by Theorem 2.4, the function $\mathfrak{n}=\mathfrak{n}_{\mathcal{U}}$ satisfies all properties of a numerosity function, plus the "if part" of (ord). Assume now that $\mathcal{U}$ is Ramsey: we have only to prove that, if $\mathfrak{n}(X)<\mathfrak{n}(Y)$, then there exists a proper subset $Z \subset Y$ such that $\mathfrak{n}(X)=\mathfrak{n}(Z)$.

By the Ramsey property of $\mathcal{U}$, every sequence of natural numbers is $\mathcal{U}$-equivalent to a nondecreasing sequence. Therefore there exists a set $\left\{n_{0}, n_{1}, \ldots\right\} \in \mathcal{U}$ such that

$$
0<\left|Y_{n_{i}}^{\alpha}\right|-\left|X_{n_{i}}^{\alpha}\right| \leq\left|Y_{n_{i+1}}^{\alpha}\right|-\left|X_{n_{i+1}}^{\alpha}\right| \text { for all } i \in \mathbb{N} \text {. }
$$

Let $Z_{0}$ be a subset of $Y_{n_{0}}$ of size $\left|X_{n_{0}}\right|$. For $i \in \mathbb{N}$ let $Z_{i+1}$ be a subset of $Y_{n_{i+1}}^{\alpha} \backslash Y_{n_{i}}^{\alpha}$ of size $\left|X_{n_{i+1}}^{\alpha}\right|-\left|X_{n_{i}}^{\alpha}\right|$. Put $Z=\bigcup_{i \in \mathbb{N}} Z_{i}$; then clearly $Z$ is a subset of $Y$ such that $\mathfrak{n}(X)=\mathfrak{n}(Z)$.

It is worth noticing that the construction above can be extended to arbitrary countable subsets of the union of all the Euclidean spaces $\bigcup_{d \in \mathbb{N}} \mathbb{E}_{d}(\mathbb{R})$.

Namely, given a countable $X \subseteq \bigcup_{d \in \mathbb{N}}\left(\mathbb{R}^{\alpha}\right)^{d}$, put

$$
X_{n}^{\alpha}=X \cap \bigcup_{d \leq n}\left(\mathbb{R}^{\alpha}\right)^{d} .
$$

The corresponding definition of the function $\mathfrak{n}_{\mathcal{F}}$ agrees with the preceding one on finite-dimensional point sets. Moreover all properties of Theorem 2.4 continue to hold, with the proviso that the product property (prod) can fail if $X$ or $Y$ contain points of arbitrarily large dimension. Similarly, the proof of Theorem 2.5 can run unchanged with the new definition of the approximations $X_{n}^{\alpha}$. Moreover, having at disposal points of arbitrary dimension, any nondecreasing sequence of natural numbers is the counting sequence $\left\langle\left|X_{n}^{\alpha}\right|\right\rangle_{n \in \mathbb{N}}$ of some set $X$.

Corollary 2.6 $(\mathrm{CH})$. Let $\mathcal{U}$ be a Ramsey ultrafilter on $\mathbb{N}$. The function

$$
\left.\mathfrak{n}_{\mathcal{U}}:\left[\bigcup_{d \in \mathbb{N}} \mathbb{E}_{d}(\mathbb{R})\right]\right]^{\leq \aleph_{0}} \rightarrow \mathbb{Z}^{\mathbb{N}}
$$

is onto $\mathbb{N}_{\mathcal{U}}^{\mathbb{N}}$ and satisfies the four properties (unit), (sum), (prod), and (ord), provided (prod) is restricted to finite-dimensional point sets. 
2.3. Measuring all point sets. Any set can be viewed as "approximable" by subsets of smaller cardinality. So, in order to also measure uncountable point sets, one might require that the numerosity function be continuous with respect to appropriately chosen approximations. With this in mind, we introduced in Definition 1.2 the notion of normal approximation of [3] and the corresponding notion of normal numerosity.

Clearly the $\omega_{1}$-sequence of countable sets $\left\langle\mathbb{R}^{\alpha} \mid \alpha<\omega_{1}\right\rangle$ is a normal approximation of $\mathbb{R}$, so $\left\langle X \cap\left(\mathbb{R}^{\alpha}\right)^{d} \mid \alpha<\omega_{1}\right\rangle$ is a normal approximation of any $d$-dimensional point set $X \subseteq \mathbb{E}_{d}(\mathbb{R})$. We shall use these approximations in order to obtain a normal numerosity function on all point sets.

If $\mathcal{V}$ is an ultrafilter on $I$ and $\pi: I \rightarrow J$ is onto, then

$$
\mathcal{U}=\pi[\mathcal{V}]=\{\pi[V] \mid V \in \mathcal{V}\}
$$

is an ultrafilter on $J$ such that the map $\left\langle x_{j}\right\rangle_{j \in J} \mapsto\left\langle x_{\pi(i)}\right\rangle_{i \in I}$ induces an isomorphic embedding $\pi_{*}: \mathbb{Z}_{\mathcal{U}}^{J} \rightarrow \mathbb{Z}_{\mathcal{V}}^{I}$ of ultrapowers (see e.g. [9]). The range of $\pi_{*}$ is the limit ultrapower $\mathbb{Z}^{I} \mathcal{V} \mid \mathcal{E}_{\pi}$, where $\mathcal{E}_{\pi}=\left\{X \subseteq I \times I \mid E_{\pi} \subseteq X\right\}$ is the filter on $I \times I$ generated by the equivalence $E_{\pi}=\left\{\left(i, i^{\prime}\right) \mid \pi(i)=\pi\left(i^{\prime}\right)\right\}$ induced by $\pi$. Recall that, by definition, if $\mathcal{E}$ is a filter on $I \times I$, then the limit ultrapower $\mathbb{Z}^{I} \mathcal{V} \mid \mathcal{E}$ contains the $\mathcal{V}$ equivalence classes of those functions $f: I \rightarrow \mathbb{Z}$ such that $\left\{\left(i, i^{\prime}\right) \mid f(i)=f\left(i^{\prime}\right)\right\} \in \mathcal{E}$.

It turns out that, given a nonprincipal ultrafilter $\mathcal{U}$ on $\mathbb{N}$, an ultrafilter $\mathcal{V}$ can be appropriately chosen so as to obtain a normal numerosity function that essentially extends the function $\mathfrak{n}_{\mathcal{U}}$ of Theorem 2.5 to the set of all (finite-dimensional) point sets. More precisely

Theorem $2.7(\mathrm{CH})$. Let

- $\mathcal{U}$ be a nonprincipal ultrafilter on $\mathbb{N}$;

- $\mathfrak{n}_{\mathcal{U}}: \mathbb{W}_{0} \rightarrow \mathbb{N}_{\mathcal{U}}^{\mathbb{N}}$ be the numerosity function defined by

$$
\mathfrak{n}_{\mathcal{U}}(X)=\left[\left\langle\left|X \cap\left(\mathbb{R}_{n}^{\alpha}\right)^{d}\right|: n \in \mathbb{N}\right\rangle\right]_{\mathcal{U}},
$$

for $X \subseteq\left(\mathbb{R}^{\alpha}\right)^{d}$;

- $\pi: \omega_{1} \rightarrow \mathbb{N}$ be the function defined by $\pi(\omega \alpha+n)=n$ for all $\alpha<\omega_{1}$;

- $\mathcal{E}_{\pi}$ be the filter on $\omega_{1} \times \omega_{1}$ generated by the equivalence induced by $\pi$ :

$$
E_{\pi}=\left\{(\omega \alpha+n, \omega \beta+n) \mid \alpha, \beta<\omega_{1}\right\} .
$$

Then there exists an ultrafilter $\mathcal{V}$ on $\omega_{1}$ such that the function $\mathfrak{n}_{\mathcal{V}}: \mathbb{W} \rightarrow \mathbb{Z}_{\mathcal{V}}^{\omega_{1}}$ mapping $X \subseteq \mathbb{E}_{d}(\mathbb{R})$ to

$$
\mathfrak{n}_{\mathcal{V}}(X)=\left[\left\langle\left|X_{\beta}\right| \mid \beta<\omega_{1}\right\rangle\right]_{\mathcal{V}} \text {, where } X_{\omega \alpha+n}=X \cap\left(\mathbb{R}_{n}^{\alpha}\right)^{d}
$$

is a normal numerosity function defined on all point sets and making the following diagram commute 8

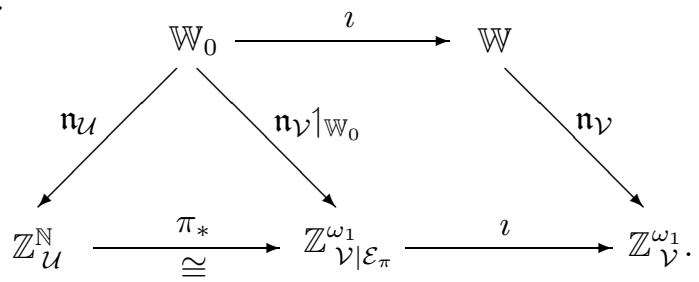

${ }^{8}$ By $\imath$ we denote the inclusion maps, and by $\mathfrak{n}_{\mathcal{V}} 1 \mathbb{w}_{0}$ we denote the restriction of $\mathfrak{n}_{\mathcal{V}}$ to the family $\mathbb{W}_{0}$ of the countable point sets. 
In particular, $\pi[\mathcal{V}]=\mathcal{U}$ and so, whenever $\mathcal{U}$ is Ramsey, $\mathfrak{n}_{\mathcal{V}}$ is a normal numerosity function defined on all point sets that is Zermelian when restricted to the countable sets.

Proof. Let us consider the following subsets of $\omega_{1}$, indexed by the family $\mathcal{F}$ of all functions $f: D \rightarrow \mathcal{U}$ with $D$ closed and unbounded in $\omega_{1}$ :

- $N(f)=\{\omega \alpha+n \mid \alpha \in \operatorname{dom} f, n \in f(\alpha)\}$ for $f \in \mathcal{F}$.

The family $\{N(f) \mid f \in \mathcal{F}\}$ has the finite intersection property. In fact, let $f_{1}, \ldots, f_{k} \in \mathcal{F}$ be given, and let $g$ be the function defined on $D=\bigcap_{i=1}^{k} \operatorname{dom} f_{i}$ by $g(\alpha)=\bigcap_{i=1}^{k} f_{i}(\alpha)$. Then clearly $g \in \mathcal{F}$ and $N(g) \subseteq \bigcap_{i=1}^{k} N\left(f_{i}\right)$.

Now let $\mathcal{V}$ be any ultrafilter on $\omega_{1}$ including all sets $N(f)$ for $f \in \mathcal{F}$. We prove that the function $\mathfrak{n}_{\mathcal{V}}$ is a normal numerosity function. In order to simplify the proof, we remark first that we may consider w.l.o.g. only pairs of point sets of the same dimension. In fact any point set $X$ of dimension $d$ has equinumerous sets in each higher dimension $d^{\prime}>d$, by definition of $\mathfrak{n}_{\mathcal{V}}$. (E.g. it suffices to reiterate $d^{\prime}-d$ many times the last component of each $d$-tuple of $X$.)

Given $\left(a_{1}, \ldots, a_{d}\right) \in \mathbb{E}_{d}(\mathbb{R})$, let $\beta, m$ be such that $a_{1}, \ldots, a_{d} \in \mathbb{R}_{m}^{\beta}$. For $\alpha>\beta$ put $f_{\beta}(\alpha)=\left\{n \in \mathbb{N} \mid n \geq \max \left(m, \varphi_{\alpha}(\beta)\right)\right\}$; then

$$
\left\{\omega \alpha+n \mid\left(a_{1}, \ldots, a_{d}\right) \in\left(\mathbb{R}_{n}^{\alpha}\right)^{d}\right\} \supseteq N\left(f_{\beta}\right),
$$

and (unit) follows. The properties (sum) and (prod) are immediate consequences of the definition of $\mathfrak{n}_{\mathcal{V}}$, which is therefore a numerosity function.

For every $z \in \mathbb{Z}^{\mathbb{N}}$, let $z^{\omega_{1}}$ be the $\omega_{1}$-sequence such that $z_{\omega \alpha+n}^{\omega_{1}}=z_{n}$ for all $\alpha<\omega_{1}$. If $\left\{n \in \mathbb{N} \mid z_{n}=w_{n}\right\}=U \in \mathcal{U}$, let $f_{U}: \omega_{1} \rightarrow \mathcal{U}$ be the constant function with value $U$. Then $z^{\omega_{1}}$ and $w^{\omega_{1}}$ agree on $N\left(f_{U}\right) \in \mathcal{V}$. Moreover $z^{\omega_{1}}$ is compatible with the equivalence $E_{\pi}$, so $\pi_{*}$ maps the $\mathcal{U}$-class of $z$ to the $\mathcal{V}$-class of $z^{\omega_{1}}$ thus providing an isomorphism $\pi_{*}: \mathbb{Z}_{\mathcal{U}}^{\mathbb{N}} \rightarrow \mathbb{Z}_{\mathcal{V} \mid \mathcal{E}_{\pi}}^{\omega_{1}}$

In order to check the commutativity of the diagram, the only nontrivial equality to prove is $\left.\mathfrak{n}_{\mathcal{V}}\right|_{\mathbb{W}_{0}}=\pi_{*} \circ \mathfrak{n}_{\mathcal{U}}$. Now, if $X$ is a countable point set, say $X \subseteq\left(\mathbb{R}^{\alpha}\right)^{d}$, put $f(\beta)=\left\{n \in \mathbb{N} \mid n \geq \varphi_{\beta}(\alpha)\right\}$ for $\alpha<\beta<\omega_{1}$. Then the $\omega_{1}$-sequences $\left\langle x_{\omega \beta+n}=\left|X \cap\left(\mathbb{R}_{n}^{\beta}\right)^{d}\right|\right\rangle_{\beta<\omega_{1}}$ and $\left\langle x_{\omega \beta+n}=\left|X \cap\left(\mathbb{R}_{n}^{\alpha}\right)^{d}\right|\right\rangle_{\beta<\omega_{1}}$ agree on $N(f) \in \mathcal{V}$. Hence $\pi_{*}\left(\mathfrak{n}_{\mathcal{U}}(X)\right)=\mathfrak{n}_{\mathcal{V}}(X)$.

Finally we prove that the numerosity function $\mathfrak{n}_{\mathcal{V}}$ is normal. Given normal approximations $\left\langle X_{\alpha} \mid \alpha<\omega_{1}\right\rangle$ and $\left\langle Y_{\alpha} \mid \alpha<\omega_{1}\right\rangle$ of the uncountable $d$-dimensional point sets $X$ and $Y$, respectively, put

$$
D=\left\{\alpha<\omega_{1} \mid X_{\alpha}=X \cap\left(\mathbb{R}^{\alpha}\right)^{d}, Y_{\alpha}=Y \cap\left(\mathbb{R}^{\alpha}\right)^{d}\right\} .
$$

Assume that $\mathfrak{n}_{\mathcal{V}}\left(X_{\alpha}\right) \leq \mathfrak{n}_{\mathcal{V}}\left(Y_{\alpha}\right)$ for all $\alpha \in D$, which is closed and unbounded in $\omega_{1}$. We claim that

$$
\left\{n \in \mathbb{N}|| X \cap\left(\mathbb{R}_{n}^{\alpha}\right)^{d}|\leq| Y \cap\left(\mathbb{R}_{n}^{\alpha}\right)^{d} \mid\right\}=U_{\alpha} \in \mathcal{U} \text { for all } \alpha \in D .
$$

Assume the contrary, and put $D_{\alpha}=D \cap\left(\omega_{1} \backslash \alpha\right)$. Define $f_{\alpha}: D_{\alpha} \rightarrow \mathcal{U}$ by

$$
f_{\alpha}(\beta)=\left\{n \in \mathbb{N} \backslash U_{\alpha} \mid n \geq \varphi_{\beta}(\alpha)\right\} \text { for all } \beta \in D_{\alpha} .
$$

Then

$$
\left\{\omega \beta+n|| X_{\alpha} \cap\left(\mathbb{R}_{n}^{\beta}\right)^{d}|>| Y_{\alpha} \cap\left(\mathbb{R}_{n}^{\beta}\right)^{d} \mid\right\} \supseteq N\left(f_{\alpha}\right) \in \mathcal{V}
$$

and so $\mathfrak{n}_{\mathcal{V}}\left(X_{\alpha}\right)>\mathfrak{n}_{\mathcal{V}}\left(Y_{\alpha}\right)$, against our assumption. 
Now define $f: D \rightarrow \mathcal{U}$ by $f(\alpha)=U_{\alpha}$. Then

$$
\left\{\omega \alpha+n|| X \cap\left(\mathbb{R}_{n}^{\alpha}\right)^{d}|\leq| Y \cap\left(\mathbb{R}_{n}^{\alpha}\right)^{d} \mid\right\} \supseteq N(f) \in \mathcal{V}
$$

and so $\mathfrak{n}_{\mathcal{V}}(X) \leq \mathfrak{n}_{\mathcal{V}}(Y)$.

The numerosity function $\mathfrak{n}_{\mathcal{V}}$ is Cantorian if and only if the ultrafilter $\mathcal{V}$ also contains the following subsets of $\omega_{1}$ :

$$
\text { - } B\left(\mathbb{R}^{\beta}, Y\right)=\left\{\omega \alpha+n|| \mathbb{R}^{\beta} \cap \mathbb{R}_{n}^{\alpha}|<| Y \cap \mathbb{R}_{n}^{\alpha} \mid\right\}
$$

for all $\beta<\omega_{1}$ and all uncountable $Y \subseteq \mathbb{R}$. The fact that this family of sets, together with the family of all sets $N(f)$ considered in the above proof, has the finite intersection property seems problematic. Observe, however, that one can always find Cantorian extensions of any given numerosity function defined on countable point sets. A construction that works with arbitrary values of the continuum is given in the next section.

\section{Dropping the Continuum Hypothesis}

In the preceding section two consequences of the Continuum Hypothesis $\mathrm{CH}$ play a crucial role. On the one hand, the reals are an increasing union of countable sets, and so we are able to define a coherent finite approximation system for all countable point sets. On the other hand, every nonprincipal filter on $\mathbb{N}$ generated by less than $\mathfrak{c}$ elements is contained in a Ramsey ultrafilter. We shall see in this section that both results can be obtained by weaker set-theoretic hypotheses, and in particular that they are relatively consistent with arbitrary values of the cardinality of the continuum.

Let $\mathcal{I}=[\mathbb{R}]^{<\aleph_{0}}$ be the set of all finite subsets of $\mathbb{R}$. For $X \subseteq \mathbb{E}_{d}(\mathbb{R})$ define the "counting $\mathcal{I}$-sequence" of $X$ by

$$
\Phi(X)=\left\langle\left|X \cap F^{d}\right| \mid F \in \mathcal{I}\right\rangle .
$$

Let $\mathcal{V}$ be an ultrafilter over $\mathcal{I}$, and consider the ultrapower $\mathbb{Z}_{\mathcal{V}}^{\mathcal{I}}$. By taking the $\mathcal{V}$ equivalence classes of the counting $\mathcal{I}$-sequences we obtain a function $\mathfrak{n}_{\mathcal{V}}: \mathbb{W} \rightarrow \mathbb{Z}_{\mathcal{V}}^{\mathcal{I}}$, where $\mathfrak{n}_{\mathcal{V}}(X)=[\Phi(X)]_{\mathcal{V}}$. It turns out that, given a nonprincipal ultrafilter $\mathcal{U}$ on $\mathbb{N}$, the ultrafilter $\mathcal{V}$ can be conveniently chosen so as to obtain a numerosity function that essentially extends the function $\mathfrak{n}_{\mathcal{U}}$ of Theorem 2.5 to all sets in $\mathbb{W}$.

In order to specify our assumptions, let us introduce the following

Definition 3.1. A function $\Psi:[\mathbb{R}]^{\aleph_{0}} \rightarrow\left([\mathbb{R}]^{<\aleph_{0}}\right)^{\mathbb{N}}$ assigning a sequence of finite subsets

$$
\Psi(X)=\left\langle X_{n} \mid n \in \mathbb{N}\right\rangle
$$

to each countable $X \subseteq \mathbb{R}$ is called a coherent finite approximation system (for $\mathbb{R}$ ) if the following conditions are fulfilled:

(a): $X_{n} \subseteq X_{n+1}$ and $\bigcup_{n \in \mathbb{N}} X_{n}=X$;

(b): if $Y \subseteq X$, then there exists $n_{X Y}$ such that $Y_{n}=X_{n} \cap Y$ for all $n \geq n_{X Y}$.

In Section 2 we assumed the Continuum Hypothesis and we defined the family of finite sets $\mathbb{R}_{n}^{\alpha}$ that directly yields a coherent finite approximation system for $\mathbb{R}$ by putting

$$
\Psi(X)=\left\langle X \cap \mathbb{R}_{n}^{\alpha} \mid n \in \mathbb{N}\right\rangle, \text { where } \alpha=\min \left\{\beta \mid X \subseteq \mathbb{R}^{\beta}\right\} .
$$

When the continuum is larger, the existence of such approximation systems for $\mathbb{R}$ is problematic. In 13, P. Koszmider investigates sufficient conditions for the existence 
of "coherent families of finite-to-one functions" on $[\kappa]]^{\aleph_{0}}$. Giving such a family amounts to a sort of "labelling" of countable sets, in the style of [1]. More precisely, the coherent family of labelling functions $\left\langle\ell_{X}: X \rightarrow \mathbb{N} \mid X \in[\kappa]^{\aleph_{0}}\right\rangle$ corresponds exactly to the coherent approximation system $\Psi(X)=\left\langle\ell_{X}^{-1}(\{0, \ldots, n\}) \mid n \in \mathbb{N}\right\rangle$. Let us write

- cas $(\kappa)$ for "there exists a coherent finite approximation system for $\kappa$ ".

Then Theorem 8 of [13] can be restated as asserting that

- $\operatorname{cas}\left(\aleph_{n}\right)$ holds for all $n \in \mathbb{N}$,

whence

Fact 3.2. If $\mathfrak{c}<\aleph_{\omega}$, then there is a coherent finite approximation system for $\mathbb{R}$.

We shall consider the status of cas $(\mathfrak{c})$ for larger values of the continuum in the final section. Here we can state our result as follows.

Theorem 3.3. Assume cas $(\mathfrak{c})$, let $\Psi$ be a coherent finite approximation system for $\mathbb{R}$, and let $\mathcal{U}$ be a nonprincipal ultrafilter on $\mathbb{N}$. Then there exists a unique numerosity function $\mathfrak{n}_{\mathcal{U}}: \mathbb{W}_{0} \rightarrow \mathbb{N}_{\mathcal{U}}^{\mathbb{N}}$ such that

$$
\mathfrak{n}_{\mathcal{U}}(Z)=\left[\left\langle\left|Z \cap Y_{n}^{d}\right| \mid n \in \mathbb{N}\right\rangle\right]_{\mathcal{U}}
$$

for all $Y \in[\mathbb{R}]^{\aleph_{0}}$ and all $Z \subseteq Y^{d}$. If the ultrafilter $\mathcal{U}$ is Ramsey, the numerosity function $\mathfrak{n}_{\mathcal{U}}$ is Zermelian.

Moreover, let $\mathcal{E}$ be the filter on $\mathcal{I} \times \mathcal{I}$ generated by the equivalences

$$
\mathcal{E}(X)=\{(F, G) \mid F \cap X=G \cap X\}
$$

for $X \in[\mathbb{R}]^{\aleph_{0}}$. Then there exist

- an ultrafilter $\mathcal{W}$ on $\mathcal{I}$;

- an isomorphism $\sigma: \mathbb{Z}_{\mathcal{U}}^{\mathbb{N}} \rightarrow \mathbb{Z}^{\mathcal{I}} \mathcal{W} \mid \mathcal{E}$

such that the function $\mathfrak{n}_{\mathcal{W}}: \mathbb{W} \rightarrow \mathbb{Z}^{\mathcal{I}}$ mapping $W \subseteq \mathbb{R}^{d}$ to

$$
\mathfrak{n}_{\mathcal{W}}(W)=\left[\left\langle\left|W \cap F^{d}\right| \mid F \in \mathcal{I}\right\rangle\right]_{\mathcal{W}}
$$

is a Cantorian numerosity function defined on all point sets and making the following diagram commute 9

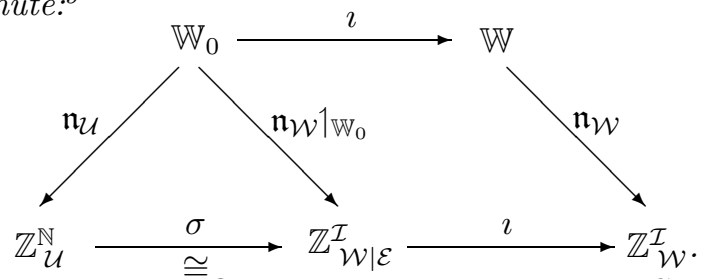

In particular, when the ultrafilter $\mathcal{U}$ is Ramsey, $\mathfrak{n}_{\mathcal{W}}$ is a Cantorian numerosity function defined on all point sets that is Zermelian when restricted to the countable sets.

Proof. We begin by proving that the definition of the map $\mathfrak{n}_{\mathcal{U}}$ is independent of the choice of $Y$. Let $X$ be another countable subset of $\mathbb{R}$ such that $Z \subseteq X^{d}$. If $n>\max \left\{n_{X \cup Y, X}, n_{X \cup Y, Y}\right\}$ then $(X \cup Y)_{n} \cap X=X_{n}$ and $(X \cup Y)_{n} \cap Y=Y_{n}$; hence

$$
Z \cap(X \cup Y)_{n}^{d}=Z \cap X_{n}^{d}=Z \cap Y_{n}^{d}
$$

\footnotetext{
${ }^{9}$ As in Theorem 2.7 by $\imath$ we denote the inclusion maps, and by $\mathfrak{n}_{\mathcal{W}} 1 \mathfrak{w}_{0}$ we denote the restriction of $\mathfrak{n}_{\mathcal{W}}$ to the family $\mathbb{W}_{0}$ of the countable point sets.
} 
So $\mathfrak{n}_{\mathcal{U}}$ is well defined, and the properties (unit), (sum), and (prod) are immediate consequences of the definition.

Assume that the ultrafilter $\mathcal{U}$ is Ramsey, and let $X, Y$ be countable $d$-dimensional point sets such that $\mathfrak{n}_{\mathcal{U}}(X)<\mathfrak{n}_{\mathcal{U}}(Y)$. Then we can pick a countable $W \subseteq \mathbb{R}$ such that $X \cup Y \subseteq W^{d}$ and repeat word by word the proof of Theorem 2.5. by simply writing $X \cap W_{n}^{d}, Y \cap W_{n}^{d}$ instead of $X_{n}^{\alpha}$ and $Y_{n}^{\alpha}$, respectively.

In order to find the ultrafilter $\mathcal{W}$, consider the following subsets of $\mathcal{I}$ :

- $\widehat{a}=\{F \in \mathcal{I} \mid a \in F\}$ for $a \in \mathbb{R}$.

- $A(X, U)=\left\{F \in \mathcal{I} \mid \exists n \in U, F \cap X=X_{n}\right\}$ for $X \in[\mathbb{R}]^{\aleph_{0}}$ and $U \in \mathcal{U}$.

- $B(Y, Z)=\left\{F \in \mathcal{I}|| F^{d} \cap Y|<| F^{d} \cap Z \mid\right\}$ for infinite $Y, Z \subseteq \mathbb{R}^{d}$ such that $|Y|<|Z|$.

We claim that all the above sets form a family $\mathcal{F} \subseteq \mathcal{P}(\mathcal{I})$ with the finite intersection property. Before proceeding, observe the following fact. Let $Y \subseteq \mathbb{R}^{d}$ and let $Y^{\prime} \subseteq \mathbb{R}^{d+1}$ be obtained from $Y$ by repeating the last component of each $d$-tuple

$$
Y^{\prime}=\left\{\left(y_{1}, \ldots, y_{d}, y_{d}\right) \mid\left(y_{1}, \ldots, y_{d}\right) \in Y\right\} .
$$

Then clearly $\left|F^{d} \cap Y\right|=\left|F^{d+1} \cap Y^{\prime}\right|$ for all $F \in \mathcal{I}$. Therefore, in proving the finite intersection property, we can assume w.l.o.g. that all the sets $Y, Z$ appearing in the considered $B(Y, Z)$ have the same dimension.

Let points $a_{1}, \ldots, a_{k} \in \mathbb{R}$, countable sets $X_{1}, \ldots, X_{k} \subseteq \mathbb{R}$, sets $U_{1}, \ldots, U_{k} \in \mathcal{U}$, and $d$-dimensional point sets $Y_{i j}$ with $\left|Y_{i j}\right|=\kappa_{i}$, for $1 \leq i, j \leq k$ and $\aleph_{0}=\kappa_{1}<$ $\kappa_{2}<\cdots<\kappa_{k}$, be given.

Pick a countable set $Z_{1} \subseteq \mathbb{R}$ such that

$$
\left\{a_{1}, \ldots, a_{k}\right\} \cup \bigcup_{i=1}^{k} X_{i} \subseteq Z_{1} \text { and } \bigcup_{j=1}^{k} Y_{1 j} \subseteq Z_{1}^{d} .
$$

Put $m=\max \left\{n_{Z_{1} X_{i}} \mid 1 \leq i \leq k\right\}$ and let

$$
U=\left\{n>m \mid n \in U_{i} \text { for } 1 \leq i \leq k\right\} \in \mathcal{U} .
$$

Pick $F_{1} \in A\left(Z_{1}, U\right)$ and let $p_{1}=\left|F_{1}^{d} \cap Z_{1}^{d}\right|$. Now pick $p_{1}+1$ elements from each $Y_{2 j} \backslash Z_{1}^{d}$, and add all their components to $F_{1}$ so as to obtain the finite set $F_{2}$. Then clearly, for all $j, j^{\prime} \leq k$,

$$
\left|F_{2}^{d} \cap Y_{1 j}\right| \leq\left|F_{2}^{d} \cap Z_{1}^{d}\right|=\left|F_{1}^{d} \cap Z_{1}^{d}\right|=p_{1}<p_{1}+1 \leq\left|F_{2}^{d} \cap Y_{2 j^{\prime}}\right| .
$$

Now pick a set $Z_{2} \supseteq Z_{1}$ of size $\kappa_{2}$ such that $\bigcup_{j=1}^{k} Y_{2 j} \subseteq Z_{2}^{d}$. Let $p_{2}=\left|F_{2}^{d} \cap Z_{2}^{d}\right|$, and pick $p_{2}+1$ elements from each $Y_{3 j} \backslash Z_{2}^{d}$, and add all their components to $F_{2}$ so as to obtain the finite set $F_{3}$. By iterating this procedure one finally gets a finite set $F_{k}$ such that for all $i<i^{\prime} \leq k$ and all $j, j^{\prime} \leq k$,

$$
\left|F_{k}^{d} \cap Y_{i j}\right|<\left|F_{k}^{d} \cap Y_{i^{\prime} j^{\prime}}\right| .
$$

Such an $F_{k}$ belongs to the intersection

$$
F_{k} \in \bigcap_{i=1}^{k} \widehat{a_{i}} \cap \bigcap_{i=1}^{k} A\left(X_{i}, U_{i}\right) \cap \bigcap_{\substack{1 \leq i<i^{\prime} \leq k \\ 1 \leq j, j^{\prime} \leq k}} B\left(Y_{i j}, Y_{i^{\prime} j^{\prime}}\right),
$$

and so the family $\mathcal{F}$ has the finite intersection property.

Now let $\mathcal{W}$ be any ultrafilter on $\mathcal{I}$ including $\mathcal{F}$. We claim that the function $\mathfrak{n}_{\mathcal{W}}$ fulfills all the properties of a Cantorian numerosity. In order to simplify the 
proof, we note that we may consider w.l.o.g. only pairs of point sets of the same dimension. In fact, if $Y \subseteq \mathbb{R}^{d}, Y^{\prime} \subseteq \mathbb{R}^{d+1}$ are as in the previous remark, then $\mathfrak{n}_{\mathcal{W}}(Y)=\mathfrak{n}_{\mathcal{W}}\left(Y^{\prime}\right)$. Now

- (unit) follows from the fact that

$$
\left\{F \in \mathcal{I}|| F^{d} \cap\left\{\left(a_{1}, \ldots, a_{d}\right)\right\} \mid=1\right\}=\widehat{a_{1}} \cap \ldots \cap \widehat{a_{d}} \in \mathcal{W} .
$$

- (sum) and (prod) are immediate consequences of the definition of $\mathfrak{n}_{\mathcal{W}}$.

- If $Y, Z$ are infinite $d$-dimensional point sets and $|Y|<|Z|$, then

$$
\left\{F \in \mathcal{I}|| F^{d} \cap X|<| F^{d} \cap Y \mid\right\}=B(Y, Z) \in \mathcal{W},
$$

and (cant) follows.

In order to define the isomorphism $\sigma$, assume w.l.o.g. that we can fix a countable $S \subseteq \mathbb{R}$ such that the approximating sequence $\left\langle S_{n}\right\rangle$ is strictly increasing.

For every $z \in \mathbb{Z}^{\mathbb{N}}$, let $z^{S}=\left\langle z_{F}^{S} \mid F \in \mathcal{I}\right\rangle$ be any $\mathcal{I}$-sequence such that $z_{F}^{S}=z_{n}$ whenever $F \cap S=S_{n}$. If $\left\{n \in \mathbb{N} \mid z_{n}=z_{n}^{\prime}\right\}=U \in \mathcal{U}$, then $z_{F}^{S}=\left(z^{\prime}\right)_{F}^{S}$ for all $F \in A(S, U) \in \mathcal{W}$. In this way, we have defined a map $\sigma: \mathbb{Z}_{\mathcal{U}}^{\mathbb{N}} \rightarrow \mathbb{Z}_{\mathcal{I}}^{\mathcal{I}}$. Observe that any $Y \supseteq S$ produces a $\mathcal{W}$-equivalent $\mathcal{I}$-sequence $z^{Y}$, because $F \cap Y=Y_{n}$ implies $F \cap S=S_{n}$ for all $n>n_{Y S}$. So the map $\sigma$ is independent of the choice of $S$.

Clearly, $\sigma$ is a ring homomorphism. If $\left\{n \in \mathbb{N} \mid z_{n} \neq 0\right\}=U \in \mathcal{U}$, then $\left\{F \in \mathcal{I} \mid z_{F}^{S} \neq 0\right\} \supseteq A(X, U) \in \mathcal{W}$; hence $\sigma$ is injective. We now show that the range of $\sigma$ is precisely $\mathbb{Z}^{\mathcal{I}} \mathcal{W}$.

Call $Y$-compatible an $\mathcal{I}$-sequence $\left\langle y_{F}\right\rangle$ such that $y_{F}=y_{G}$ whenever $F \cap Y=$ $G \cap Y$. Any element of the limit ultrapower $\mathbb{Z}_{\mathcal{I} \mid \mathcal{E}}^{\mathcal{I}}$ is the $\mathcal{W}$-equivalence class of an $\mathcal{I}$-sequence $\left\langle y_{F}\right\rangle$ which is $Y$-compatible for some countable $Y \subseteq \mathbb{R}$. In fact, let $Y^{(i)}$ be countable subsets of $\mathbb{R}$ for $1 \leq i \leq k$, and let $Y$ be their union. Let

$$
U=\left\{n \in \mathbb{N} \mid n>n_{Y Y^{(i)}} \text { for } 1 \leq i \leq k\right\} \in \mathcal{U} .
$$

Then

$$
A(Y, U)^{2} \cap \mathcal{E}(Y)=A(Y, U)^{2} \cap \bigcap_{i=1}^{k} \mathcal{E}\left(Y^{(i)}\right)
$$

and so any $\mathcal{I}$-sequence that respects the equivalence $\bigcap_{i=1}^{k} \mathcal{E}\left(Y^{(i)}\right)$ is $\mathcal{W}$-equivalent to a $Y$-compatible $\mathcal{I}$-sequence.

Let $y=\left\langle y_{F}\right\rangle \in \mathbb{Z}_{\mathcal{W} \mid \mathcal{E}}^{\mathcal{I}}$ be $Y$-compatible. Let $z=\left\langle z_{n}\right\rangle$ be the sequence such that $z_{n}=y_{Y_{n}}$. Put

$$
U^{\prime}=\left\{n \in \mathbb{N} \mid n>\max \left\{n_{S \cup Y, S}, n_{S \cup Y, Y}\right\}\right\} \in \mathcal{U}
$$

then $z_{F}^{S}=y_{F}$ for all $F \in A\left(S \cup Y, U^{\prime}\right) \in \mathcal{W}$, and so the $\mathcal{W}$-equivalence class of $y$ is the image under $\sigma$ of the $\mathcal{U}$-equivalence class of $z$.

Notice that if $\pi: \mathcal{I} \rightarrow \mathbb{N}$ is any function such that $\pi(F)=n$ whenever $F \cap S=S_{n}$, then $\sigma=\pi_{*}$, and so $\sigma$ is an isomorphism of ultrapowers.

We are left with the commutativity of the diagram, which reduces to proving that $\sigma\left(\mathfrak{n}_{\mathcal{U}}(Z)\right)=\mathfrak{n}_{\mathcal{W}}(Z)$ for all $Z \in \mathbb{W}_{0}$. Let $Z \subseteq Y^{d}$, for some countable $Y \supseteq S$; then a representative of $\mathfrak{n}_{\mathcal{U}}(Z)$ is the sequence $z=\left\langle\left|\left(Y_{n}\right)^{d} \cap Z\right|\right\rangle_{n \in \mathbb{N}}$. Hence a representative of $\sigma\left(\mathfrak{n}_{\mathcal{U}}(Z)\right)$ is any $\mathcal{I}$-sequence $\left\langle z_{F}^{Y} \mid F \in \mathcal{I}\right\rangle$ such that $z_{F}^{Y}=\left|\left(Y_{n}\right)^{d} \cap Z\right|$ whenever 
$F \cap Y=Y_{n}$. On the other hand, a representative of $\mathfrak{n}_{\mathcal{W}}(Z)$ is the $\mathcal{I}$-sequence $\left\langle\left|F^{d} \cap Z\right| \mid F \in \mathcal{I}\right\rangle$, which agrees with the preceding one for all $F \in A(Y, \mathbb{N})$.

The proof is now complete.

Unfortunately the Cantorian numerosity function $\mathfrak{n}_{\mathcal{W}}$ need not be normal. In order to obtain normal numerosity functions extending $\mathfrak{n}_{\mathcal{U}}$, let us remark that the proof of Theorem 2.7 can be generalized to the case $\mathfrak{c}=\aleph_{n}$. In fact one can directly perform the first $n$ steps of the construction used in the proof of Theorem 2.2 of [3], and obtain

Theorem 3.4. Assume that $\mathfrak{c}<\aleph_{\omega}$, let a coherent finite approximation system for $\mathbb{R}$ be fixed, and let $\mathcal{U}$ be a nonprincipal ultrafilter on $\mathbb{N}$.

Let $\mathfrak{n}_{\mathcal{U}}: \mathbb{W}_{0} \rightarrow \mathbb{N}_{\mathcal{U}}^{\mathbb{N}}$ be the numerosity function such that

$$
\mathfrak{n}_{\mathcal{U}}(Z)=\left[\left\langle\left|Z \cap Y_{n}^{d}\right| \mid n \in \mathbb{N}\right\rangle\right]_{\mathcal{U}}
$$

for all $Y \in[\mathbb{R}]^{\aleph_{0}}$ and all $Z \subseteq Y^{d}$.

Let $\pi: \mathfrak{c} \rightarrow \mathbb{N}$ be the natural projection modulo $\omega$, i.e.

$$
\pi(\omega \alpha+n)=n \text { for all } \alpha<\mathfrak{c} .
$$

Then there exist

- an ultrafilter $\mathcal{V}$ on $\mathfrak{c}$ such that $\pi[\mathcal{V}]=\mathcal{U}$;

- a normal numerosity function $\mathfrak{n}_{\mathcal{V}}: \mathbb{W} \rightarrow \mathbb{Z}^{\mathfrak{c}}$ defined on all point sets and making the following diagram commute 10

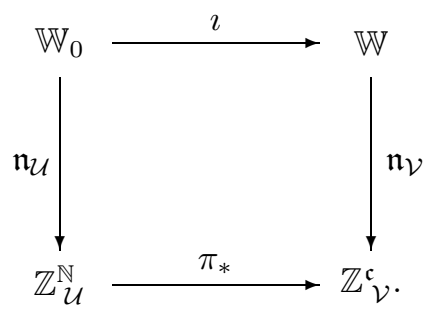

In particular, when the ultrafilter $\mathcal{U}$ is Ramsey, $\mathfrak{n}_{\mathcal{V}}$ is a normal numerosity function defined on all point sets that is Zermelian when restricted to the countable sets.

In view of Fact 3.2 , the Main Theorem of Section 1 is completely proved by combining Theorems 3.3 and 3.4 above.

\section{Final REMARKS AND OPEN QUeSTiOns}

In the previous sections we obtained numerosity functions that are Zermelian on countable point sets by assuming the existence of Ramsey ultrafilters on $\mathbb{N}$. This assumption is unavoidable if one wants to make use of the counting sequences associated to countable sets when a coherent finite approximation system is given. In fact the function $\mathfrak{n}$ of Corollary 2.6] is Zermelian if and only if the ultrafilter $\mathcal{U}$ is Ramsey, and also in Theorem 2.5 the ultrafilter $\mathcal{U}$ has to be a special $P$-point (see [5]). The existence of ultrafilters playing the corresponding role for normal approximations of uncountable sets seems to be problematic, even assuming $\mathrm{CH}$. For instance, in the notation of Theorem 2.7 in order to obtain that the function

\footnotetext{
${ }^{10}$ As in Theorem 2.7 we denote by $\imath$ the inclusion map, and by $\pi_{*}$ the canonical embedding mapping the $\mathcal{U}$-class of the sequence $z$ to the $\mathcal{V}$-class of the $\mathfrak{c}$-sequence $z \circ \pi$.
} 
$\mathfrak{n}_{\mathcal{V}}$ is Zermelian, the ultrafilter $\mathcal{V}$ should be chosen so as to satisfy the following condition:

- for all $X, Y \subseteq \mathbb{R}$ there exists $Z \subseteq \mathbb{R}$ such that

$$
\left\{\beta<\omega_{1}||\left|X_{\beta}\right|-\left|Y_{\beta}\right||=| Z_{\beta} \mid\right\} \in \mathcal{V} .
$$

Observe that this condition cannot be satisfied in general by $\omega_{1}$-sequences $x_{\beta}, y_{\beta}$, even when the corresponding countable numerosities $x^{\alpha}=\left[\left\langle x_{\omega \alpha+n}\right\rangle\right]_{\mathcal{U}} \in \mathbb{N}_{\mathcal{U}}^{\mathbb{N}}$ and $y^{\alpha}=\left[\left\langle y_{\omega \alpha+n}\right\rangle\right]_{\mathcal{U}} \in \mathbb{N}_{\mathcal{U}}^{\mathbb{N}}$ are nondecreasing in $\alpha$. E.g. take $y^{\alpha}=x^{\alpha}+a^{\alpha}$, where $\left\langle a^{\alpha}\right\rangle$ is a suitable $\omega_{1}$-sequence coinitial in the infinites of $\mathbb{N}_{\mathcal{U}}^{\mathbb{N}}$.

Similarly, if the numerosity $\mathfrak{n}_{\mathcal{W}}$ of Theorem 3.3 has to be Zermelian, then the ultrafilter $\mathcal{W}$ has to fulfill the very demanding condition

- for all $X, Y \subseteq \mathbb{R}$ there exists $Z \subseteq \mathbb{R}$ such that

$$
\{F \in \mathcal{I}|\| X \cap F|-|Y \cap F||=| Z \cap F \mid\} \in \mathcal{W} .
$$

As pointed out at the end of Section 2 the task of obtaining numerosity functions that are simultaneously Cantorian and normal also seems to require the use of very special ultrafilters. Even assuming the Continuum Hypothesis, in the notation of Theorem 2.7 the function $\mathfrak{n}_{\mathcal{V}}$ is Cantorian if and only if the ultrafilter $\mathcal{V}$ satisfies the following condition:

- for all $\beta<\omega_{1}$ and all uncountable $Y \subseteq \mathbb{R}$,

$$
\left\{\omega \alpha+n|| \mathbb{R}^{\beta} \cap \mathbb{R}_{n}^{\alpha}|<| Y \cap \mathbb{R}_{n}^{\alpha} \mid\right\} \in \mathcal{V} .
$$

In Section 3 we assumed cas $(\mathfrak{c})$ and constructed Cantorian numerosities extending a given numerosity function for countable sets. It is worth noticing that the methods of [2] would suffice to obtain Cantorian numerosities in ZFC alone. But then the countable numerosities would be elements of the larger ultrapower $\mathbb{N}_{\mathcal{W}}^{\mathcal{I}}$, and so, as shown above, no familiar set-theoretic hypothesis would ensure the existence of an ultrafilter $\mathcal{W}$ making them Zermelian. So the essential use of cas (c) was that of allowing for countable numerosities that are elements of a "small" set of hypernatural numbers, namely an ultrapower $\mathbb{N}_{\mathcal{U}}^{\mathbb{N}}$, which are Zermelian whenever the ultrafilter $\mathcal{U}$ is Ramsey.

The existence of Ramsey ultrafilters is independent of ZFC. However, many "light" sufficient conditions for their existence can be given, which allow for an arbitrary size of the continuum. E.g., if $\operatorname{cov}(\mathcal{B})=\mathfrak{c}$, then every filter generated by less than $\mathfrak{c}$ sets is contained in a Ramsey ultrafilter. Moreover the addition of uncountably many Cohen reals ensures $\operatorname{cov}(\mathcal{B})=\mathfrak{c}$ (see 4, 9.26 and 11.3). Hence the equality $\operatorname{cov}(\mathcal{B})=\mathfrak{c}$ is consistent with arbitrary values of $\mathfrak{c}$.

Concerning the hypothesis cas $(\mathfrak{c})$ per se, the arguments in the second section of [13] can be put together so as to prove the following.

Theorem 4.1 ([13]). Let $\kappa$ be a cardinal of uncountable cofinality, and suppose that for every uncountable cardinal of countable cofinality $\lambda<\kappa$ the following holds:

- $\square_{\lambda}+$ there exists a cofinal family of size $\lambda^{+}$in $[\lambda]^{\aleph_{0}}$.

Then cas $(\kappa)$ holds. In particular,

$$
V=L \Longrightarrow \forall \kappa \operatorname{cas}(\kappa) .
$$


The above theorem immediately yields the Fact 3.2 that $\mathfrak{c}<\aleph_{\omega}$ implies cas $(\mathfrak{c})$. When the continuum is larger, the question is open as to whether cas $(\mathfrak{c})$ is provable in ZFC alone. ( $\square_{\aleph_{\omega}}$ suffices if $\mathfrak{c}=\aleph_{\omega+1}$, by Theorem 4.1)

Observe however that if $M[G]$ is a proper forcing extension of $M$, then $\left([\kappa]^{\aleph_{0}}\right)^{M}$ is cofinal in $\left([\kappa]^{\aleph_{0}}\right)^{M[G]}$ (see e.g. [12], Lemma 31.5). As a consequence any coherent finite approximation system for $\kappa$ in $M$ can be extended to $M[G]$ by intersection. So by adding $\kappa$ many Cohen reals to a model of $V=L$, we get the following consistency result:

Corollary 4.2. Let $\kappa$ be a cardinal of uncountable cofinality in a model $M$ of $V=L$, and let $G$ be generic for the Cohen forcing adding $\kappa$ many reals to $M$. Then

$$
M[G] \models \operatorname{cov}(\mathcal{B})=\mathfrak{c}=\kappa+\operatorname{cas}(\mathfrak{c}) .
$$

In particular, the existence of a Cantorian numerosity function defined on all point sets and Zermelian on countable sets is relatively consistent with arbitrary sizes of the continuum.

We conclude the paper by listing a few open questions that we consider the most interesting ones in this field of research.

(1) Is the existence of Zermelian numerosity functions defined on all point sets consistent with ZFC?

(2) Is it possible to have numerosity functions defined on all point sets, which are simultaneously normal and Cantorian?

(3) Is the existence of normal numerosity functions defined on all point sets provable in ZFC, or at least consistent with an arbitrary size of the continuum?

\section{REFERENCES}

1. V. Benci, M. Di Nasso - Numerosities of labelled sets: A new way of counting, Adv. Math. 173 (2003), 50-67. MR.1954455 (2004b:03065)

2. V. Benci, M. Di Nasso, M. Forti - An Aristotelian notion of size, Ann. Pure Appl. Logic 143 (2006), 43-53. MR2258620 (2007g:03070)

3. V. Benci, M. Di NAsso, M. ForTi - An Euclidean measure of size for mathematical universes, Logique et Analyse 50 No.197 (2007). MR2308603 (2008d:03057)

4. A. BLASS - Combinatorial cardinal characteristics of the continuum, in Handbook of Set Theory (M. Foreman, M. Magidor, and A. Kanamori, eds.), to appear.

5. A. Blass, M. Di NAsso, M. Forti - Quasi-selective ultrafilters and countable numerosities, in preparation.

6. G. Cantor - Mitteilungen zur Lehre vom Transfiniten, Zeitschr. Philos. philos. Kritik 91 (1887), 81-125; 92 (1888), 240-265.

7. G. Cantor - Beiträge zur Begründung der transfiniten Mengenlehre, Math Annalen 46 (1895), 481-512; 49 (1897), 207-246. MR1510964

8. G. CANTOR - Gesammelte Abhandlungen mathematischen und philosophischen Inhalts (E. Zermelo, ed.), Berlin, 1932 (reprinted 1990).

9. C.C. Chang, H.J. KeIsler - Model Theory (3rd edition), North-Holland, Amsterdam, 1990. MR.1059055 (91c:03026)

10. J.W. Dauben - Georg Cantor: His Mathematics and Philosophy of the Infinite, Princeton University Press, (reprint) 1990. MR1082146 (91h:01044)

11. EuClid - The Elements, T.L. Heath (translator), 2nd edition (reprint), Dover, New York, 1956. MR0075873(17:814b) 
12. T. JECH - Set Theory (3rd edition), Springer, Berlin, 2003. MR 1940513 (2004g:03071)

13. P. Koszmider - On coherent families of finite-to-one functions, J. Symb. Logic 58 (1993), 128-138. MR1217181 (94d:03100)

Dipartimento di Matematica "L. Tonelli", Università di Pisa, Pisa, Italy

E-mail address: dinasso@dm.unipi.it

Dipartimento di Matematica Applicata "U. Dini", Università di Pisa, Pisa, Italy

E-mail address: forti@dma.unipi.it 